\title{
XÁC ĐịNH HÀM LƯợNG MỘT SỐ ACID SIALIC TRONG THỰC PHẨM BẢO VẸ SỨC KHỎE BẦNG KỸ THUẬT SẮC KÝ LỎNG KHỐI PHỔ HAI LẦN (LC-MS/MS)
}

Nguyễn Thị Hồng Ngọc ${ }^{*}$, Mạc Thị Thanh Hoa ${ }^{1}$, Dương Thị Mai Hoa ${ }^{1}$, Trần Hùng Sơn ${ }^{1}$, Cao Công Khánh ${ }^{1}$, Phạm Thị Thanh Hà ${ }^{1}$ Lê Thị Hồng Hảo ${ }^{1}$

${ }^{1}$ Viện Kiểm nghiệm an toàn vệ sinh thưc phẩm quốc qua

${ }^{2}$ Trường Đại học Dược Hà Nội

(Ngày đến tòa soạn: 2/7/2019; Ngày sủa bài sau phản biện: 10/9/2019;

Ngày chấp nhận đăng: 18/9/2019)

\section{Tóm tắt}

Phương pháp định lượng đồng thời một số hoạt chất nhóm acid sialic (N-acetyl neuraminic acid - NANA và N-glycolyl neuraminic acid - NGNA) bằng kỹ thuật sắc ký lỏng khối phổ hai lần (LC-MS/MS) đã được nghiên cứu và khảo sát trên nền mẫu thực phẩm bảo vệ sức khỏe (TPBVSK). Quá trình phân tách được thực hiện với cột $\mathrm{C} 18(75 \mathrm{~mm}$ x 3,9 mm, 2,5 $\mu \mathrm{m})$ và pha động đẳng dòng gồm acid formic $0,1 \%$ : acetonitrile $(80: 20, \mathrm{v} / \mathrm{v})$. Các acid sialic trong tự nhiên tồn tại ở dạng liên hợp được thủy phân bằng acid formic $1 \mathrm{M}$ ở $80^{\circ} \mathrm{C}$ trong 2 giờ trước khi phân tích trên thiết bị LC-MS/MS. Kết quả thẩm định cho thấy phương pháp có độ đặc hiệu và độ chọn lọc tốt, đường chuẩn tuyến tính trong khoảng $0,04-4,25 \mu \mathrm{g} / \mathrm{ml}$, độ lặp lại và độ thu hồi đáp ứng được yêu cầu phân tích theo AOAC. Phương pháp đã được áp dụng để phân tích 45 mẫu TPBVSK trên thị trường.

Tù̀ khóa: Acid sialic, $N$-acetyl neuraminic acid, NANA, N-glycolyl neuraminic acid, NGNA, thực phẩm bảo vệ súc khỏe, TPBVSK.

\section{1. ĐĂT VẤN ĐỀ}

Acid sialic là một nhóm gồm khoảng 60 hoạt chất có bản chất đường, là dẫn xuất của neuraminic acid. Các dẫn xuất đã được biết đến như dẫn xuất phosphate hóa, dẫn xuất sulfate hóa hoặc bổ sung thêm nhiều nhóm thế lactyl và acetyl. Acid sialic có mặt tại cơ và dịch thể của tất cả các động vật có xương sống (dịch não tủy, nước bọt, dịch vị, huyết thanh, nước mắt, nước tiểu và sữa), trứng cá và thậm chí trên màng cầu khuẩn gây viêm màng não. Mật độ acid sialic cao nhất được tìm thấy ở hệ thần kinh trung ương, chủ yếu là màng tế bào thần kinh, ước tính cao hơn 20 lần so với mật độ ở màng tế bào thường với chức năng chủ yếu là tăng độ nhạy cảm giữa các tế bào [1], [2].

Mọi cơ thể động vật sống đều có thể sản sinh acid sialic, nhưng không có nghĩa là lượng sialic sinh ra đủ để đáp ứng những nhu cầu tất yếu của cơ thể. Lượng tiêu thụ acid sialic nhiều hay ít phụ thuộc vào nhiều yếu tố như: đối tượng đặc biệt (trẻ em, phụ nữ mang thai và cho con bú, người già) và trạng thái bệnh lý. Do vậy, sử dụng bổ sung acid sialic thông qua chế độ dinh dưỡng có thể góp phần duy trì lượng acid sialic ổn định cho các chức năng nội sinh quan trọng hoạt động bình thường [3], [4].

Trong hầu hết các loại sản phẩm có nguồn gốc động vật, các acid sialic chủ yếu là hỗn hợp của $\mathrm{N}$-acetyl neuraminic acid (NANA) và N-glycolyl neuraminic acid (NGNA). Tuy NANA và NGNA có mặt ở tất cả các động vật xương sống nhưng riêng NGNA lại không xuất hiện ở cơ thể người do sự mất đoạn gen chuyển hóa từ NANA thành NGNA [4].

*Điện thoại:0975565542 Email: hngoc1710@gmail.com 
Hiện nay ở Việt Nam chưa có nhiều nghiên cứu xác định đồng thời các acid sialic trong thực phẩm bảo vệ sức khỏe (TPBVSK), gây khó khăn cho việc quản lý và giám sát chất lượng các sản phẩm có chứa nhóm chất này. Do đó, nhằm góp phần bảo vệ quyền lợi người tiêu dùng cũng như hỗ trợ các cơ quan chức năng trong việc kiểm soát tốt vấn đề an toàn và chất lượng thực phẩm, nghiên cứu này sẽ khảo sát, lựa chọn và đưa ra phương pháp phân tích đồng thời hàm lượng một số hoạt chất nhóm acid sialic (gồm NANA và NGNA) trong sản phẩm TPBVSK. Trong đó, phương pháp sắc ký lỏng khối phổ được sử dụng với ưu điểm độ nhạy và độ chính xác cao.

\section{2. ĐỐI TƯỢNG VÀ PHƯƠNG PHÁP NGHIÊN CƯU}

\section{1. Đối tượng nghiên cứu}

- Đối tượng phân tích: NANA và NGNA;

- Đối tượng mẫu: TPBVSK (dạng sữa bột, dạng sữa lỏng và nước yến).

\subsection{Phương pháp nghiên cứu}

2.2.1. Thiết bị, hóa chất

\subsubsection{Thiết bị, dụng cu}

- Hệ thống sắc ký lỏng siêu hiệu năng (UPLC) Acquity gồm bơm cao áp, bộ điều nhiệt cột, bộ tiêm mẫu tự động kết nối với detector khối phổ của hãng Waters;

- Cột Xbridge C18 (75 mm x 4,6mm, 3,5 m) và tiền cột tương ứng (Waters);

- Các dụng cụ và thiết bị phụ trợ khác trong phòng thí nghiệm.

\subsubsection{Hóa chất, chất chuẩn}

- Chất chuẩn $\mathrm{N}$-acetyl neuraminic acid (Sigma);

- Chất chuẩn N-glycolyl neuraminic acid (Sigma);

- Acetontrile (Merck);

- Acid formic (Merck);

- Nước đề ion;

- Các hóa chất khác sử dụng trong nghiên cứu đều thuộc loại tinh khiết phân tích (Merck, Sigma).

\subsubsection{Nội dung nghiên cúu}

- Khảo sát và lựa chọn các điều kiện phân tích (điều khiện khối phổ và điều kiện thủy phân);

- Thẩm định phương pháp;

- Áp dụng phân tích hàm lượng NANA và NGNA trong một số mẫu thực tế.

\section{KẾT QUẢ VÀ BÀN LUẬN}

\subsection{Nghiên cứu xây dựng phương pháp phân tích}

\subsubsection{Khảo sát và lự chọn điều kiện sắc ký}

Qua tham khảo các tài liệu [9], [10], kết hợp với tiến hành khảo sát trên thiết bị UPLC-Acquity Xevo TQD, điều kiện sắc ký để xác định đồng thời NANA và NGNA được lựa chọn như sau:

- Cột Xbridge BEH (75mm x 3,9mm, 2,5 ㅆm) và tiền cột tương ứng;

- Pha động: acid formic 0,1 \%: acetonitrile (80:20);

- Tốc độ dòng: $0,2 \mathrm{~mL} /$ phút;

- Thể tích tiêm mẫu: $10 \mu \mathrm{L}$.

Điều kiện khối phổ được được tối ưu tự động bằng phần mềm tích hợp với thiết bị, cho kết quả 
NANA và NGNA đều phân tích bằng chế độ ESI (-). Sắc đồ phân tích đồng thời NANA và NGNA tại điều kiện tối ưu được thể hiện trong hình 1.

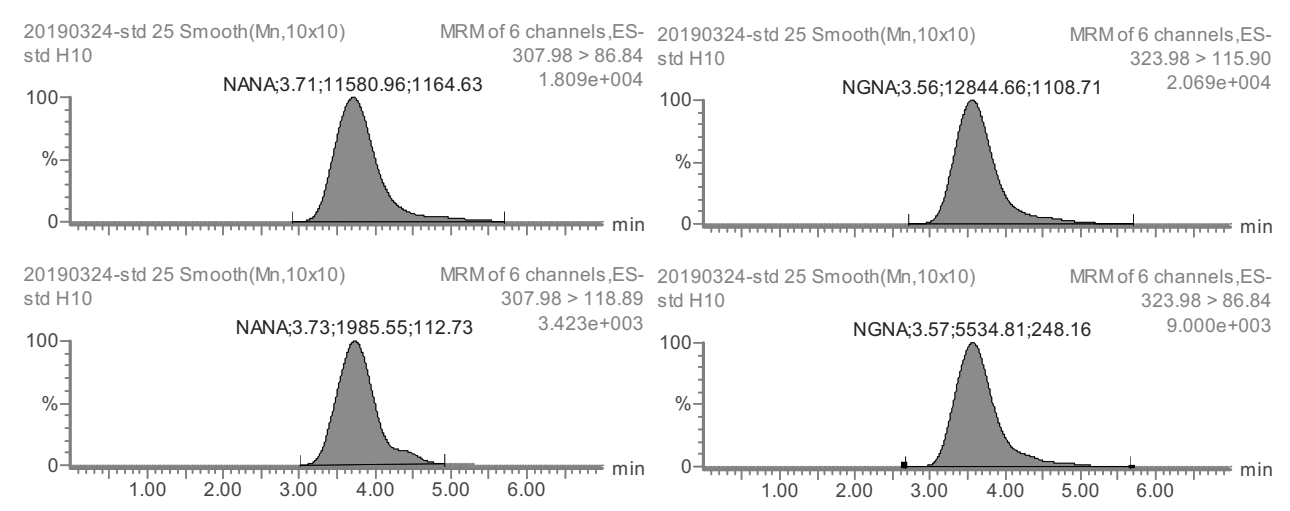

Hình 1. Sắc đồ NANA và NGNA tại điều kiện phân tích tối uu

Do công thức cấu tạo dễ dàng mất đi một $\mathrm{H}^{+}$, nên có thể phân mảnh ion ở chế độ ion âm. Tuy nhiên, không phải thiết bị nào cũng cho độ nhạy cần thiết với các ion âm. Vì vậy, một vài nghiên cứu đã sử dụng dẫn xuất để khóa nhóm $\mathrm{R}_{1}$ trong công thức cấu tạo, tăng tín hiệu tại nhóm amin để tạo thành ion dương. Tuy nhiên, việc lựa chọn ion âm có tính chọn lọc tốt hơn, do số lượng chất phân tích cho ion âm là rất ít so với ion dương, hoàn toàn phù hợp và đáp ứng yêu cầu phân tích, đặc biệt trên thiết bị sử dụng là detector khối phổ ba tứ cực. Do đó, nghiên cứu đã tiến hành trên chế độ ion âm cho kết quả phân mảnh tương thích với các nghiên cứu trước đây $[9,10]$.

\subsubsection{Khảo sát và lựa chọn điều kiện xử lý mẫu}

Trong tự nhiên, NANA và NGNA thường tồn tại ở dạng liên hợp trong các chuỗi glycan trên bề mặt tế bào, vì vậy cần thủy phân để chuyển về dạng tự do nhằm thuận lợi cho quá trình phân tích bằng LC-MS/MS.

Việc giải phóng acid sialic khỏi dạng liên hợp của chúng có thể được thực hiện bằng cách thủy phân với acid hoặc với enzyme. Mặc dù thủy phân acid có chi phí thấp và khá hiệu quả, nhưng rất khó kiểm soát được sự cân bằng phù hợp giữa giải phóng hoàn toàn và bắt đầu phân hủy chất phân tích. Nghiên cứu của Schauer đã chứng minh thủy phân mẫu với acid sulfuric $50 \mathrm{mM}$ hoặc acid hydrochloric $100 \mathrm{mM}\left(50\right.$ phút, $\left.80^{\circ} \mathrm{C}\right)$ có thể dẫn đến phân hủy lên tới $10 \%$ [11]. Tương tự, acid chlorohydric $25 \mathrm{mM}$, acid trifluoroacetic $25 \mathrm{mM}$ (TFA) hoặc acid acetic $2 \mathrm{M}\left(2\right.$ giờ, $\left.80^{\circ} \mathrm{C}\right)$ có thể gây giảm hiệu suất tới $20 \%$ [12]. Theo Lamari và Karamanos [13] và Schauer [11], acid formic gây ra sự phá hủy ít hơn, nhưng quá trình thủy phân có thể không hoàn toàn. Điều này đã dẫn đến một số lượng lớn các điều kiện thủy phân khác nhau được nghiên cứu để giải phóng acid sialic. Phương pháp thủy phân bằng enzyme ít có khả năng phân hủy acid sialic tự do, ngay cả khi kéo dài thời gian phân tích [14]. Tuy nhiên, do đặc tính chọn lọc, một loại enzyme chỉ đặc trưng cho một vài acid sialic nên phương pháp này có chi phí cao hơn [15].

Để phù hợp với điều kiện phòng thí nghiệm, và tham khảo các tài liệu nêu trên, acid formic đã được lựa chọn là tác nhân thủy phân, với các thông số khảo sát là nồng độ, nhiệt độ và thời gian tiến hành thí nghiệm.

\section{- Khảo sát nồng độ dung dịch acid formic}

Trong các nghiên cứu trước đây $[13,14]$, một số tác nhân thường được sử dụng để thủy phân NANA và NGNA từ dạng liên hợp thành dạng tự do, bao gồm acid formic, acid chlorohydric, acid sulfuric và acid trifloroacetic. Trong đó, acid formic đã được lựa chọn để khảo sát trong nghiên cứu do tính chất không tạo cặn muối không tan gây nhiễm bẩn hệ thống. 
Kết quả khảo sát nồng độ acid formic ảnh hưởng đến tín hiệu chất phân tích được trình bày trong hình 2 .

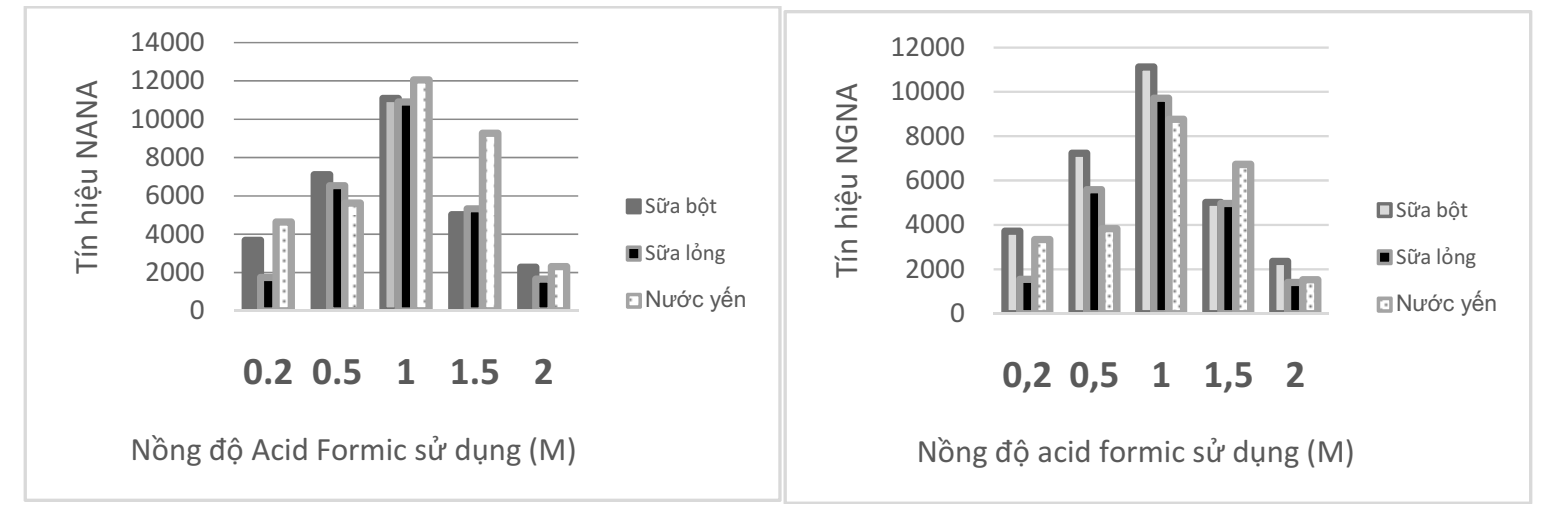

Hình 2. Anh hương của nồng độ dung dịch acid formic đến tín hiệu NANA và NGNA

Các kết quả cho thấy rằng NANA và NGNA đều có tín hiệu cao nhất ở acid formic $1 \mathrm{M}$. Ở nồng độ acid thấp hơn có thể quá trình thủy phân chưa xảy ra hoàn toàn, nếu nồng độ acid cao hơn có thể dẫn đến sự phân hủy của chất phân tích. Do đó acid formic với nồng độ $1 \mathrm{M}$ được lựa chọn cho các khảo sát tiếp theo.

- Khảo sát nhiệt độ và thời gian thủy phân

Kết quả khảo sát ảnh hưởng của nhiệt độ trong khoảng $60^{\circ} \mathrm{C}-100^{\circ} \mathrm{C}$ đến hiệu suất thủy phân cho thấy tín hiệu của NANA và NGNA tăng dần khi tăng nhiệt độ từ $60^{\circ} \mathrm{C}$ đến $80^{\circ} \mathrm{C}$. Khoảng từ $80^{\circ} \mathrm{C}-90^{\circ} \mathrm{C}$, tín hiệu chất phân tích cao nhất và hằng định. Từ $90^{\circ} \mathrm{C}$, tín hiệu chất phân tích giảm nhanh, có thể do NANA và NGNA dạng tự do sau giải phóng đã bị phân hủy ở nhiệt độ cao.

Trong thí nghiệm về thời gian, tín hiệu của NANA và NGNA cũng tăng dần và đạt trị số hằng định sau 120 phút. Quá trình thủy phân có thể chưa xảy ra hoàn toàn trong thời gian ngắn hơn. Tuy nhiên, thủy phân trong thời gian dài hơn tuy không gây phân hủy chất phân tích nhưng sẽ tốn thời gian không cần thiết.

Như vậy, trên cơ sở các kết quả khảo sát, điều kiện thủy phân được lựa chọn trong nghiên cứu này là ở $80^{\circ} \mathrm{C}$ trong 2 giờ với acid formic $1 \mathrm{M}$.

\subsection{Xác nhận giá trị sử dụng của phương pháp phân tích}

Phương pháp tối ưu được lựa chọn trên cơ sở các điều kiện phân tích và thủy phân, được dùng để xác nhận giá trị sử dụng theo các yêu cầu của $\mathrm{AOAC}$ về thẩm định phương pháp hóa học. Kết quả thẩm định được thể hiện trong bảng 1 .

Bảng 1. Kết quả thẩm định NANA và $N G N A$

\begin{tabular}{|c|c|c|c|c|}
\hline Nền mẫu & $\begin{array}{l}\text { Chất phân } \\
\text { tích }\end{array}$ & TPBVSK bột & TPBVSK lỏng & Nuớc yến \\
\hline \multirow{2}{*}{$\begin{array}{c}\text { Độ phù hợp của hệ } \\
\text { thống }\end{array}$} & NANA & \multicolumn{3}{|c|}{$\mathrm{RSD}_{\text {tín hiệu }}=1,99 \% ; \mathrm{RSD}_{\mathrm{tR}}=0,17 \%(<2,0 \%)$} \\
\hline & NGNA & \multicolumn{3}{|c|}{$\mathrm{RSD}_{\text {tín hiệu }}=1,29 \% ; \mathrm{RSD}_{\mathrm{tR}}=0,15 \%(<2,0 \%)$} \\
\hline Độ đặc hiệu & \multicolumn{4}{|c|}{$\begin{aligned} \mathrm{IP}= & 4(1 \text { ion mẹ }+2 \text { ion con }) \\
& \text { Tỷ lệ ion đạt } \pm 20 \%\end{aligned}$} \\
\hline \multirow{2}{*}{ Khoảng tuyến tính } & NANA & \multicolumn{3}{|c|}{0,04 dến $4,25 \mu \mathrm{g} / \mathrm{mL}$} \\
\hline & NGNA & \multicolumn{3}{|c|}{0,04 đến $3,80 \mu \mathrm{g} / \mathrm{mL}$} \\
\hline
\end{tabular}

6 | Tạp chí KIỂM NGHIỆM VÀ AN TOÀN THỰC PHẨM (Số 3-2019) 


\begin{tabular}{|c|c|c|c|c|}
\hline \multirow{2}{*}{ Nền mẫu } & $\begin{array}{c}\text { Chất phân } \\
\text { tích }\end{array}$ & TPBVSK bột & TPBVSK lỏng & Nước yến \\
\hline \multirow{2}{*}{$\begin{array}{c}\text { Giới hạn phát hiện } \\
\text { (LOD) }(\boldsymbol{m g} / \mathbf{k g}, \boldsymbol{L})\end{array}$} & NANA & 0,68 & 0,02 & 0,03 \\
\cline { 2 - 5 } & NGNA & 0,59 & 0,02 & 0,02 \\
\hline \multirow{2}{*}{$\begin{array}{c}\text { Giới hạn định luộng } \\
\text { (LOQ) }(\boldsymbol{m g} / \mathbf{k g}, \boldsymbol{L})\end{array}$} & NANA & 2,26 & 0,08 & 0,10 \\
\cline { 2 - 5 } & NGNA & 1,98 & 0,06 & 0,07 \\
\hline \multirow{2}{*}{ \#ộ lặp lại (RSD \%) } & NANA & 1,08 & 4,00 & 2,80 \\
\cline { 2 - 5 } & NGNA & 2,40 & 2,00 & 2,20 \\
\hline \multirow{2}{*}{ Độ thu hồi $(\boldsymbol{R} \%)$} & NANA & $97,3-100,7$ & $97,3-106,9$ & $92,3-102,7$ \\
\cline { 2 - 5 } & NGNA & $95,3-104,6$ & $93,6-105,2$ & $95,8-105,7$ \\
\hline
\end{tabular}

Phương pháp đã thẩm định và chứng minh sự phù hợp theo các tiêu chí của hướng dẫn $\mathrm{AOAC}$ về thẩm định phương pháp hóa học.

Đề cập đến các thông số thẩm định với các nghiên cứu trước đây cũng sử dụng chế độ ESI-, LOD đạt được trong nghiên cứu này (Bảng 1) tốt hơn so với một số nghiên cứu trước $[13,14,16]$.

\subsection{Kết quả xác định hàm lượng NANA và NGNA trên một số mẫu TPBVSK}

Áp dụng phương pháp đã xây dựng và thẩm định để kiểm nghiệm các mẫu TPBVSK được lấy ngẫu nhiên trên thị trường, chủ yếu tập trung vào TPBVSK sử dụng cho đối tượng trẻ sơ sinh, thanh thiếu niên trong độ tuổi phát triển và những người đang trong chế độ dinh dưỡng đặc biệt.

Hàm lượng NANA và NGNA trong một số mẫu thực tế được thể hiện trong hình 3.

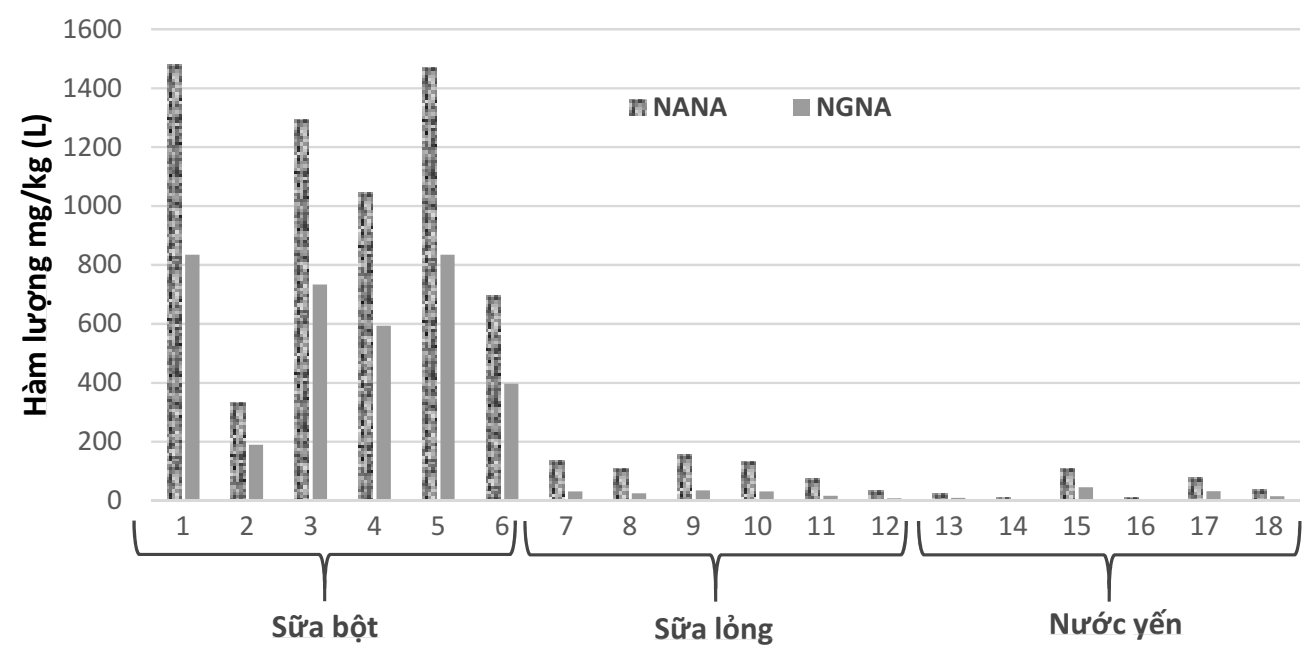

Hình 3. Hàm lượng NANA và NGNA trong một số mẫu thực tế

Qua khảo sát sơ bộ 45 mẫu TPBVSK trên thị trường thấy hàm lượng NANA và NGNA dao động trong khoảng từ $4-1800 \mathrm{mg} / \mathrm{kg}(\mathrm{L})$, trong đó có 15 mẫu dạng sữa bột, 15 mẫu dạng sữa lỏng và 15 mẫu nước yến. Hàm lượng NANA và NGNA đa dạng phụ thuộc chủ yếu vào tỷ lệ công thức pha chế và nguồn gốc của nguyên liệu sialic acid.

\section{KẾT LUẠN}

Phương pháp đã được tối ưu và thẩm định đạt theo yêu cầu ISO/IEC 17025.

Phương pháp có thể áp dụng để phân tích TPBVSK có chứa NANA và NGNA nói riêng và acid 
sialic nói chung trên thị trường. Với hóa chất thông dụng, thời gian phân tích trên thiết bị ngắn (7 phút), đã được thẩm định theo tiêu chí AOAC, phương pháp có thể dễ dàng được chuyển giao cho các phòng thí nghiệm có thiết bị sắc ký lỏng khối phổ. Phương pháp có đủ điều kiện tiến hành thẩm định liên phòng thí nghiệm để phát triển thành phương pháp chính thức, góp phần khẳng định chất lượng các sản phẩm có chứa acid sialic trên thị trường.

\section{TÀI LIẸU THAM KHẢO}

1. Arne Lundblad (2015), "Gunnar Blix and his discovery of sialic acids. Fascinating molecules in glycobiology”, Upsala Journal of Medical Sciences, 120, pp. 104-112.

2. Leonard W., Herbert F. (1962), "The Biosynthesis of Sialic Acids”, The Journal of Biological Chemistry, 237(5), pp. 1421-1431.

3. Christoph H. Röhrig, Sharon S. H. Choi \& Nigel Baldwin (2017), "The Nutritional Role of Free Sialic Acid, a Human Milk Monosaccharide, and Its Application as a Functional Food Ingredient", Critical Reviews in Food Science and Nutrition, 57(5), pp. 1017-1038.

4. Brendon D. Gill, Harvey E. Indyk, David C. Woollard (2016), "Current Methods for the Analysis of Selected Novel Nutrients in Infrant Formulas and Adult Nutritionals", Journal of AOAC International, 99, pp. 30-41.

5. Ajit Varki (2008),"Sialic acids in human health and disease", Trends in Molecular Medicine, 14(8), pp. 351-360.

6. Wang, B., Brand-Miller, J., Mcveagh, P. \& Petocz, P. (2001): "Concentration and distribution of sialic acid in human milk and infant formulas", American Journal of Clinical Nutrition, 74, pp. 510-515.

7. Svennerholm, L., Bostrom, K., Jungbjer, B. \& Olsson, L. (1994): “Membrane lipids of adult human brain: lipid composition of frontal and temporal lobe in subjects of age 20 to 100 years", Journal of Neurochemicals, 63, pp. 1802-1811.

8. AOAC (2013), “Appendix K: Guidelines for Dietary Supplements and Botanicals” AOAC official methods of analysis.

9. Dan Wang, Xiang Zhou, Lin Wang, Sihe Wang, Xue-Long Sun (2014), "Quantification of free sialic acid in human plasma through a robustquinoxalinone derivatization and LC-MS/MS using isotope-labeledstandard calibration", Journal of Chromatography B, 994, pp. $75-81$.

10. D.Karunanithi, A.Radhakrishna \& V.M.Biju (2013), "Quantitative Determination of Sialic Acid in Indian Milk and Milk Products", International Journal of Applied Biology and Pharmaceutical Technology, 4(1), pp. 318-323.

11. R. Schauer (1982): "Chemistry, metabolism, and biological functions of sialic acids", Advances in Carbohydrate Chemistry and Biochemistry, 40, pp. 131-234.

12. N.K. Karamanos, B. Wikstrom, C.A. Antonopoulos, A. Hjerpe (1990): "Determination of $\mathrm{N}$-acetyl- and N-glycolylneuraminic acids in glycoconjugates by reversed phase high-performance liquid chromatography with ultra-violet detection", Journal of Chromatography A 503, pp. 421-429.

13. F.N. Lamari, N.K. Karamanos (2002): "Separation methods for sialic acids and critical evaluation of their biologic relevance", Journal of Chromatography B, 781, pp. 3-19.

14. T. Hikita, K. Tadano-Aritomi, N. Iida-Tanaka, H. Toyoda, A. Suzuki, T. Toida, T. Imanari, T. Abe, Y. Yanagawa, I. Ishizuka (2000): "Determination of N-acetyl- and N-glycolyl 
neuraminic acids in gangliosides by combination of neuraminidase hydrolysis and fluorometric high-performance liquid chromatography using a GM3 derivative as an internal standard", Analytical of Biochemistry, 281, pp. 193-201.

15. G. Reuter, R. Schauer (1994): "Determination of sialic acids", Methods in Enzymology, 230, pp. 168-199.

16. Fredoen Valianpour, Nicolaas G.G.M. Abeling, Marinus Duran, Jan G.M. Huijmans \& Willem Kulik (2004), "Quantification of Free Sialic Acid in Urine by HPLC-Electrospray Tandem Mass Spectrometry: A Tool for the Diagnosis of Sialic Acid Storage Disease", Clinical Chemistry, 50(2), pp. 403-409.

\section{Summary}

\section{DETERMINATION OF SIALIC ACID IN DIETARY SUPPLEMENT BY LIQUID CHROMATOGRAPHY TANDEM-MASS SPECTROMETRY (LC-MS/MS)}

Nguyen Thi Hong Ngoc ${ }^{1}$, Mac Thi Thanh Hoa ${ }^{1}$, Duong Thi Mai Hoa ${ }^{1}$, Tran Hung Son ${ }^{1}$, Cao Cong Khanh', Pham Thi Thanh Ha ${ }^{2}$, Le Thi Hong Hao ${ }^{1}$

\section{${ }^{1}$ National Institute for Food Control \\ ${ }^{2}$ Hanoi University of Pharmacy}

Simultaneous determination of sialic acids (N-acetyl neuraminic acid - NANA and $\mathrm{N}$-glycolyl neuraminic acid - NGNA) in dietary supplement matrices by liquid chromatography tandem-mass spectrometry (LC-MS/MS) had been developed. Sialic acids were separated by using a C18 column ( $75 \mathrm{~mm} \times 3.9 \mathrm{~mm}, 2.5 \mu \mathrm{m}$ ) and isocratic mobile phase of acid formic $0.1 \%$ : acetonitrile $(80: 20, \mathrm{v} / \mathrm{v})$. Sialic acids were totally hydrolyzed by formic acid $1 \mathrm{M}$ at $80^{\circ} \mathrm{C}$ in 2 hours before being analyzed by LC-MS/MS. It was proved that the method with the linearity range of $0.04-4.25 \mu \mathrm{g} / \mathrm{ml}$ had highly specificity, selectivity, repeatability, and recovery rate, that well satisfied AOAC requirements. The method has been applied to detect sialic acids in commercial dietary supplements.

Keywords: Sialic acid, $N$-acetyl neuraminic acid, NANA, $N$-glycolyl neuraminic acid, NGNA, dietary supplement. 